\title{
Subspace Hamiltonian technique
}

\author{
Roland E. Allen and Madhu Menon \\ Department of Physics, Texas A\&M University, College Station, Texas 77843-4242
}

(Received 22 July 1985)

\begin{abstract}
The subspace Hamiltonian technique, introduced several years ago, has proved convenient for determining bound states and resonances associated with surfaces and defects. Here we discuss the extension of this technique to other problems, including total-energy calculations and real-time computer simulations. We prove several theorems and report numerical calculations that help to explain the usefulness of the technique in practical applications.
\end{abstract}

\section{INTRODUCTION}

Green's-function methods are useful for at least two reasons: (1) They often allow an $N$-dimensional problem to be reduced to an $n$-dimensional one, where $N$ is large (or infinite) and $n$ is small (or at least finite). (2) They allow many-body effects to be included.

Several years ago, within the general context of the Green's-function approach, one of us introduced a "subspace Hamiltonian" technique. ${ }^{1}$ This technique has proved convenient for calculating the bound states and resonances associated with surfaces, ${ }^{2}$ interfaces, ${ }^{3,4}$ and defects. ${ }^{5}$ Here we extend the technique to other problems, including total-energy calculations and real-time computer simulations. ${ }^{6}$ We prove several theorems and report numerical calculations that help explain the utility of this technique.

\section{THE SUBSPACE HAMILTONIAN}

The subspace Hamiltonian is an energy-dependent effective Hamiltonian. ${ }^{1}$ (For concreteness, we will consider the one-electron Hamiltonian for electrons in a solid. The treatment of this paper also applies when many-body effects are included, with $H \rightarrow H+\Sigma-\mu$, where $\Sigma$ is the self-energy and $\mu$ the chemical potential. ${ }^{7}$ It also applies when $H \rightarrow D$, where $D$ is the dynamical matrix for phonons.) If $\overleftrightarrow{\mathrm{H}}$ is the $N \times N$ Hamiltonian matrix for a "large" system (e.g., an infinite solid), the Green's function for this system is

$$
\overleftrightarrow{\mathrm{G}}(E) \equiv(E \overleftrightarrow{\mathrm{1}}-\overleftrightarrow{\mathrm{H}})^{-1} \text {, }
$$

where $\overleftrightarrow{1}$ is the $N \times N$ identity matrix. Let some $n$ dimensional subspace be chosen, and the rows and columns permuted so that $i, j=1,2, \ldots, n$ within this subspace. We partition $\overleftrightarrow{\mathrm{G}}$ in the well-known fashion:

$$
\overleftrightarrow{\mathrm{G}}=\left(\begin{array}{ll}
G^{11} & G^{12} \\
G^{21} & G^{22}
\end{array}\right),
$$

where $G^{11}, G^{12}, G^{21}$, and $G^{22}$ are, respectively, $n \times n$, $n \times(N-n),(N-n) \times n$, and $(N-n) \times(N-n)$. That is, $G^{11}$ is the Green's function in the chosen subspace. For simplicity of notation, we write

$$
G=G^{11} \text {. }
$$

We then let

$$
E-H_{\text {sub }}(E) \equiv[G(E)]^{-1}
$$

The subspace Hamiltonian $H_{\text {sub }}$ is only $n \times n$, whereas the original Hamiltonian $\overleftrightarrow{\mathrm{H}}$ is $N \times N$. The price that we pay for this reduction is that $H_{\text {sub }}$ is energy dependent. In addition, as will be seen below, it is frequently nonHermitian. The main point of this paper is to show that the replacement of $\overleftrightarrow{\mathrm{H}}$ by $H_{\text {sub }}(E)$ is very advantageous despite these complications. We will also see that the subspace Hamiltonian technique has advantages over more conventional Green's-function methods.

The eigenvalues $E_{i}$ and $n$-dimensional eigenvectors $\psi_{i}$ are also energy dependent:

$$
H_{\text {sub }}(E) \psi_{i}(E)=E_{i}(E) \psi_{i}(E) \text {. }
$$

Suppose that

$$
\overleftrightarrow{\mathbf{H}}=\overleftrightarrow{\mathbf{H}}_{0}+\overleftrightarrow{\mathrm{V}}
$$

where $\overleftrightarrow{\mathrm{H}}_{0}$ is an unperturbed Hamiltonian and $\overleftrightarrow{\mathrm{V}}$ has nonzero elements only in some $n \times n$ subspace:

$$
\overleftrightarrow{\mathrm{V}}=\left(\begin{array}{ll}
V & 0 \\
0 & 0
\end{array}\right) .
$$

With the partitioning of (2.2), the one-electron $N \times N$ Dyson equation

$$
\begin{aligned}
& \overleftrightarrow{\mathrm{G}}=\overleftrightarrow{\mathrm{G}}_{0}+\overleftrightarrow{\mathrm{G}}_{0} \cdot \overleftrightarrow{\mathrm{V}} \cdot \overleftrightarrow{\mathrm{G}} \\
& \overleftrightarrow{\mathrm{G}}_{0}(E) \equiv\left(E \stackrel{\overrightarrow{1}}{-}-\overleftrightarrow{\mathrm{H}}_{0}\right)^{-1}
\end{aligned}
$$

is essentially equivalent to the $n \times n$ Dyson equation

$$
G=G_{0}+G_{0} V G
$$

as is quite well known. Since (2.10) is equivalent to

$$
G^{-1}=G_{0}^{-1}-V
$$

(2.4) is equivalent to

$$
E-H_{\text {sub }}(E)=G_{0}^{-1}-V .
$$




\section{THEOREM 1: MONOTONIC DECREASE OF $E_{i}(E)$}

We now prove that

$$
\frac{\partial E_{i}(E)}{\partial E} \leqq 0
$$

whenever the energy $E$ lies within a band gap-i.e., outside the continua of unbound states. In surface-state calculations, or other problems where there is twodimensional translational invariance, we define $G(\overline{\mathbf{k}} E)$, $E_{i}(\overline{\mathrm{k}} E)$, etc., where $\overline{\mathrm{k}}=\left(k_{i}, k_{2}\right)$ is the planar wave vector. Then (3.1) is valid within the band gaps at fixed $\bar{k}$, which cover a larger range of energies than the intersection of the band gaps for all $\bar{k}$. Furthermore, (3.1) is a good approximation in energy regions where the bulk density of states is low.

The significance of (3.1) is that it limits the behavior of the eigenvalues $E_{i}(E)$ of the subspace Hamiltonian. They cannot vary wildly and erratically within the band gaps because (3.1) guarantees they will decrease monotonically. One consequently expects, and finds in numerical calculations, that $E_{i}(E)$ is a smooth function of $E$ within the band gaps.

To prove (3.1), we differentiate $(2.12)$ with respect to $E$ :

$$
\frac{\partial H_{\text {sub }}(E)}{\partial E}=1+G_{0}^{-1} \frac{\partial G_{0}}{\partial E} G_{0}^{-1}
$$

since $V$ is independent of $E$. (In the present section we do not consider many-body effects.) According to the Hellmann-Feynman theorem,

$$
\frac{\partial E_{i}(E)}{\partial E}=\bar{\psi}_{i}^{\dagger}(E) \frac{\partial H_{\mathrm{sub}}(E)}{\partial E} \psi_{i}(E),
$$

where

$$
H_{\mathrm{sub}}^{\dagger}(E) \bar{\psi}_{i}(E)=E_{i}^{*}(E) \bar{\psi}_{i}(E)
$$

At this point we are still allowing $G(E)$ and $H_{\text {sub }}(E)$ to be non-Hermitian, so that the right eigenvectors $\psi_{i}$ of (2.5) and the left eigenvectors $\bar{\psi}_{i}^{\dagger}$ of (3.4) must be distinguished, and $E_{i}$ is, in general, complex. Inner products are, of course, implied in equations like (2.5), (3.4), and (3.3). Equation (3.3) follows from

$$
E_{i}(E)=\bar{\psi}_{i}^{\dagger}(E) H_{\mathrm{sub}}(E) \psi_{i}(E)
$$

and the normalization condition

$$
\bar{\psi}_{i}^{\dagger}(E) \psi_{i}(E)=1
$$

[We assume the normal case that the eigenvectors of $H_{\text {sub }}$ can be made to satisfy (3.6).] From (3.2) and (3.3) we obtain

$$
\frac{\partial E_{i}}{\partial E}=1+\bar{\psi}_{i}^{\dagger}(E) G_{0}^{-1} \frac{\partial G_{0}}{\partial E} G_{0}^{-1} \psi_{i}(E) .
$$

Equation (2.1) implies the well-known relation

$$
\frac{\partial}{\partial E} \overleftrightarrow{\mathrm{G}}_{0}=-\overleftrightarrow{\mathrm{G}}_{0}^{2}
$$

(Recall that $\overleftrightarrow{\mathrm{G}}_{0}$ is $N \times N$ and $G_{0}$ is $n \times n$.) The partitioning of (2.2) then yields

$$
\frac{\partial}{\partial E} G_{0}=-G_{0}^{2}-G_{0}^{12} G_{0}^{21}
$$

so that (3.2) and (3.7), respectively, become

$$
\begin{aligned}
& \frac{\partial H_{\mathrm{sub}}(E)}{\partial E}=-\left(G_{0}^{11}\right)^{-1} G_{0}^{12} G_{0}^{21}\left(G_{0}^{11}\right)^{-1} \\
& \frac{\partial E_{i}(E)}{\partial E}=-\bar{\psi}_{i}^{\dagger}(E)\left(G_{0}^{11}\right)^{-1} G_{0}^{12} G_{0}^{21}\left(G_{0}^{11}\right)^{-1} \psi_{i}(E) .
\end{aligned}
$$

In these last equations, we have reverted to writing $G_{0}^{11}$ for $G_{0}$. Notice that the energy dependence of $H_{\text {sub }}$ and the $E_{i}$ arises from the coupling of the subspace 1 to the external space 2 via the Green's functions $G_{0}^{12}$ and $G_{0}^{21}$. If this coupling is turned off, $H_{\text {sub }}$ and the $E_{i}$ become constant with respect to variations in the energy $E$.

For energies lying within the bulk bands,

$$
E \rightarrow E+i \delta
$$

in (2.1), as usual (in the case of the retarded Green's function). Then $\overleftrightarrow{\mathrm{G}}$ is not Hermitian. Let us now, however, restrict our attention to real energies outside the bulk bands, where $\overleftrightarrow{\mathrm{G}}_{0}, \overleftrightarrow{\mathrm{G}}$, and $H_{\text {sub }}$ are Hermitian. Then (3.10) becomes

$$
\begin{aligned}
& \frac{\partial H_{\mathrm{sub}}(E)}{\partial E}=-\left[G_{0}^{21}\left(G_{0}^{11}\right)^{-1}\right]^{\dagger}\left[G_{0}^{21}\left(G_{0}^{11}\right)^{-1}\right], \\
& \frac{\partial E_{i}(E)}{\partial E}=-\left[G_{0}^{21}\left(G_{0}^{11}\right)^{-1} \psi_{i}(E)\right]^{\dagger}\left[G_{0}^{21}\left(G_{0}^{11}\right)^{-1} \psi_{i}(E)\right] .
\end{aligned}
$$

Since the right-hand side of $(3.12 b)$ has the form $-|\Psi|^{2}$, (3.1) follows immediately.

Notice that we could have used $G$ rather than $G_{0}$ in the derivation (3.2)-(3.12). That is, (3.1) is true in general [so long as $G(E)$ is Hermitian], and not just for systems described by (2.7).

\section{THEOREM 2: $H_{\text {sub }}(E) \rightarrow H$ as $|E| \rightarrow \infty$}

In this section we prove that

$$
H_{\text {sub }}(E) \rightarrow H \text { as }|E| \rightarrow \infty,
$$

where

$$
\overleftrightarrow{\mathrm{H}}=\left(\begin{array}{ll}
H^{11} & H^{12} \\
H^{21} & H^{22}
\end{array}\right)
$$

and

$$
H=H^{11} \text {, }
$$

just as in (2.2) and (2.3). This theorem provides an even more important limitation on the behavior of $H_{\text {sub }}$, its eigenvalues $E_{i}(E)$, and its eigenvectors $\psi_{i}(E)$, since it implies that they are all constant with respect to the energy $E$ 
at large $E$. This allows a very great simplification in applications of the subspace Hamiltonian technique, as will be discussed in Sec. VI. Equation (4.1) is equivalent to the statement that the subspace 1 is decoupled from the external space 2 at large energies.

To prove (4.1), we expand (2.1):

$$
\overleftrightarrow{\mathrm{G}}(E)=E^{-1}\left(\overleftrightarrow{1}+\overleftrightarrow{\mathrm{H}} / E+\overleftrightarrow{\mathrm{H}}^{2} / E^{2}+\cdots\right) \text {. }
$$

Within the subspace 1 of $(4.2)$, this is

$$
G(E)=E^{-1}\left(1+H / E+H^{2} / E^{2}+H^{12} H^{21} / E^{2}+\cdots\right) .
$$

Then (2.4) gives

$E-H_{\text {sub }}(E)=E\left(1-H / E-H^{12} H^{21} / E^{2}+\cdots\right)$,

with (4.1) following immediately.

\section{THEOREM 3: BOUND STATES AND RESONANCES CORRESPOND TO $E_{i}(E)=E$}

We now prove that the Green's function diverges at those energies $E$ for which

$$
E_{i}(E)=E \text {. }
$$

A solution to (5.1) outside the continua of unbound states will ordinarily correspond to a bound state if $E$ is real or a resonance if $E$ is complex.

Equation (5.1) provides a useful method to search for bound states and resonances in calculations-a much better method, in our opinion, than the traditional approach of looking for solutions to

$$
\operatorname{det}\left[1-G_{0}(E) V\right]=0 \text {. }
$$

In the first place, Eq. (5.1) focuses on individual eigenvalues whose behavior is relatively simple. Equation (5.2), on the other hand, focuses on a determinant that may involve the product of many eigenvalues, and whose behavior may consequently be very complicated. In the second place, (5.1) is more physically meaningful than (5.2), in that (5.2) can be rewritten as

$$
\operatorname{det} G_{0}(E) \operatorname{det}\left[E-H_{\text {sub }}(E)\right]=0 .
$$

Solutions to (5.2) thus correspond to both the physical bound states and resonances of (5.1), and to the unphysical solutions of $\operatorname{det} G_{0}(E)=0$.

To prove (5.1), we merely recognize that, at fixed energy $E, G$ is given by the usual spectral representation:

$$
G(E)=\sum_{i} \frac{\psi_{i}(E) \bar{\psi}_{i}^{\dagger}(E)}{E-E_{i}(E)}
$$

We are assuming the normal case that the eigenvectors of $H_{\text {sub }}$ satisfy the completeness relation

$$
\sum_{i} \psi_{i}(E) \bar{\psi}^{\dagger}(E)=1
$$

so that (5.4) follows from (2.4) and (2.5). Then $G(E)$ diverges at those energies $E$ satisfying (5.1).
For reasonably narrow resonances, a convenient approximation to $(5.1)$ is

$$
\operatorname{Re} E_{i}(E)=E \text {. }
$$

This criterion has yielded surface-state resonances that are in agreement with experiment for the (110) surfaces of $\mathrm{ZnSe}$ and $\mathrm{GaAs}^{2}$ In $\mathrm{Si} / \mathrm{NiSi}_{2}$ interface-state calculations, ${ }^{4}$ it has also been found to agree with the alternative theoretical criterion that a resonance corresponds to a peak in the local density of states,

$$
\rho_{\mathrm{loc}}(\bar{k} E)=-\frac{1}{\pi} \operatorname{Im} \operatorname{Tr} G(\overline{\mathrm{k}} E)
$$

\section{CALCULATING THE TOTAL ENERGY AND OTHER PHYSICAL QUANTITIES}

As mentioned in the preceding section, bound states correspond to poles $E$ along the real axis and resonances correspond to poles out in the complex plane. However, these resonance poles are not on the physical Riemann sheet. Instead they lie on unphysical Riemann sheets. ${ }^{8}$ In numerical calculations, one finds that

$$
\operatorname{Im} E_{i}(E) \lesseqgtr 0 \text { for } \operatorname{Im} E \gtrless 0
$$

for input energies $E$ on the physical sheet. That is, the singularities (5.1) for $\operatorname{Im} E \neq 0$ are not found on the physical sheet. This is a fortunate fact, because it implies that, according to Cauchy's theorem, one can deform contours of integration involving the Green's function.

As an illustration, consider

$$
\Delta E=\int_{-\infty}^{E_{F}} E \Delta \rho(E) d E,
$$

where $\Delta \rho(E)$ is the change in the density of states produced by the perturbation $V$. This is one contribution to the change in total energy; other contributions, and changes in other physical quantities, will involve ideas similar to those discussed below. According to the theorem of DeWitt and Toulouse, 9,10

$$
\Delta \rho(E)=\frac{1}{\pi} \frac{\partial \eta(E)}{\partial E}
$$

where $\eta(E)$ is the phase shift:

$$
\eta(E) \equiv-\operatorname{Im} \ln \operatorname{det}\left(G^{-1} G_{0}\right) .
$$

If we restrict our attention to neutral systems, for which the Friedel sum rule ${ }^{11,12}$ holds,

$$
\eta\left(E_{F}\right)=0 \text {, }
$$

with the usual convention $\eta(-\infty) \equiv 0$, integration by parts gives

$$
\Delta E=\frac{-1}{\pi} \int_{E_{c}}^{E_{F}} \eta(E) d E .
$$

Here, $E_{c}$ is some energy below all the relevant states, so that $\eta(E)=0$ for $E<E_{c}$.

Equations (2.4) and (2.5) allow (6.4) to be rewritten as

$$
\eta(E)=-\operatorname{Im}\left[\sum_{i} \ln \left\{\left[E-E_{i}(E)\right] /\left[E-E_{i}^{(0)}(E)\right]\right\}\right),
$$


where

$$
\begin{aligned}
& H_{\mathrm{sub}}^{(0)}(E) \psi_{i}^{(0)}(E)=E_{i}^{(0)}(E) \psi_{i}^{(0)}(E), \\
& E-H_{\mathrm{sub}}^{(0)}(E) \equiv\left[G_{0}(E)\right]^{-1}
\end{aligned}
$$

Equation (4.1) implies that

$$
\begin{array}{r}
\eta(E) \approx-\sum_{i} \operatorname{Im}\left\{\ln \left[\left(E-\varepsilon_{i}\right) /\left(E-\varepsilon_{i}^{(0)}\right)\right]\right\} \\
\text { for |E | large, }
\end{array}
$$

where the $\varepsilon_{i}$ are the eigenvalues of the actual Hamiltonian $H$ that the subspace would have if it were decoupled from the external space. [See (4.2) and (4.3). Recall that $H$ is $n \times n$, whereas $\overleftrightarrow{\mathrm{H}}$ is $N \times N$.] Similarly, the $\varepsilon_{i}^{(0)}$ are the eigenvalues of $H_{0}$, with

$$
H=H_{0}+V \text {. }
$$

According to the argument in the first paragraph of this section, we are allowed to deform the contour of (6.6):

$$
\Delta E=\frac{-1}{\pi} \int_{C} \eta(E) d E
$$

where $C$ begins at some real energy $E_{c}$ well below the band energies of interest, follows a path in the complex plane above the real axis and well removed from these band energies, and then descends from some complex energy to the real axis at the Fermi energy $E_{F}$. For example, $C$ may consist of the straight line segments $\left(E_{c}, 0\right)$ to $\left(E_{c}, E_{b}\right),\left(E_{c}, E_{b}\right)$, to $\left(E_{F}, E_{b}\right)$, and $\left(E_{F}, E_{b}\right)$ to $\left(E_{F}, 0\right)$. According to (4.1), (6.10) will be a good approximation for all energies on $C$, except those on the final part of the final segment as $E$ descends to $E_{F}$.

If $C_{1}$ is the large contour along which (6.10) is a good approximation-i.e., along which

$$
E_{i}(E) \approx E_{i}\left(E_{0}\right)
$$

with $E_{0}$ being some fixed complex energy lying on $C_{1}-$ and if $C_{2}$ is the small contour along which the energy dependence of $E_{i}(E)$ cannot be neglected, then

$$
\begin{aligned}
& \Delta E=\Delta E_{1}+\Delta E_{2}, \\
& \Delta E_{1} \approx \frac{1}{\pi} \operatorname{Im}\left[\sum _ { i } \int _ { C _ { 1 } } \left\{\ln \left[E-E_{i}\left(E_{0}\right)\right]\right.\right. \\
& \left.\left.\quad-\ln \left[E-E_{i}^{(0)}\left(E_{0}\right)\right]\right\} d E\right),
\end{aligned}
$$

$$
\begin{aligned}
\Delta E_{2}=\frac{1}{\pi} \operatorname{Im}\left[\sum _ { i } \int _ { C _ { 2 } } \left\{\ln \left[E-E_{i}(E)\right]\right.\right. & \\
& \left.\left.\quad-\ln \left[E-E_{i}^{(0)}(E)\right]\right\} d E\right) .
\end{aligned}
$$

Integrals like (6.15a) are relatively simple and in some cases can even be done analytically. Computationally, $(6.15 a)$ and $(6.15 b)$ represent an enormous reduction of the original problem (6.2). In numerical calculations for an $s p^{3} s^{*}$ model of semiconductors, ${ }^{13}$ we find that an accurate calculation of $\Delta E$ from (6.2) requires $\sim 100$ energies, whereas the same level of accuracy is obtained from (6.15) with $\sim 5$ energies_one energy $E_{0}$ on $C_{1}$ and about four on $C_{2} \cdot{ }^{14}$ In parallel studies, Sankey ${ }^{15}$ has obtained about $1 \%$ accuracy in a calculation of $\Delta E$ that employed two energies on a contour that we would characterize as $C_{1}$ and three on a contour that corresponds to our $C_{2}$.

In Figs. 1-4 we display the rather slow dependence of the eigenvalues $E_{i}(E)$ on energies $E$ in the complex plane. Notice that $E_{i}(E)$ is indeed almost constant until one returns to the Fermi energy $E_{F}$ on the real axis. Figs. 1 and 2 , in particular, demonstrate that $(6.15)$ is a useful approximation, with $C_{1}$ corresponding to most of the rectangular contour of Figs. 1 and 2, and $C_{2}$ corresponding to the extreme right-hand side of these figures.

Other physical quantities are represented by other integrals involving Green's functions. In general, however, the original integrals along the real axis should be deformed into the complex plane. Along the part of the contour well removed from the relevant bands $\left(C_{1}\right)$, we use a representation like (5.4) for the Green's function, with the approximation (6.13) and

$$
\psi_{i}(E) \approx \psi_{i}\left(E_{0}\right), \quad \bar{\psi}_{i}(E) \approx \bar{\psi}_{i}\left(E_{0}\right)
$$

On the other hand, along that part of the contour that represents a return to the real axis at the Fermi energy $E_{F}$

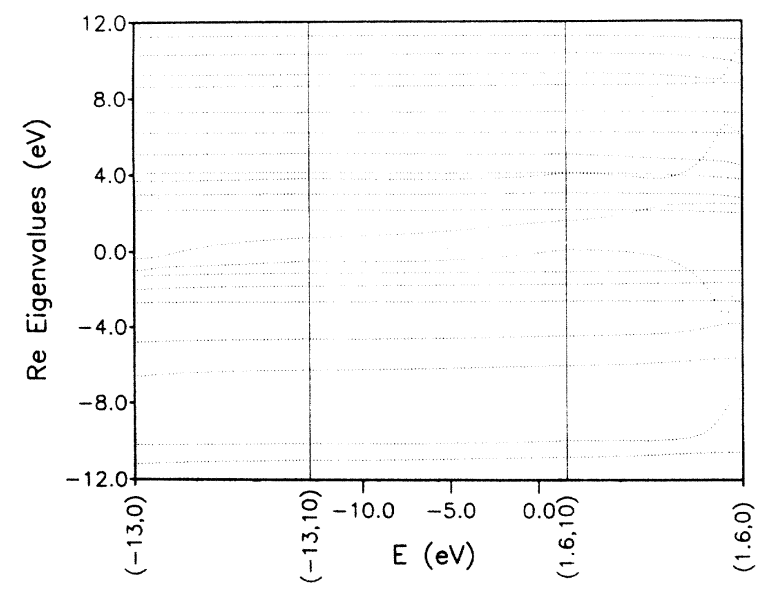

FIG. 1. $\operatorname{Re} E_{i}(E)$ for energies $E$ in the complex plane. Here, $H_{\text {sub }}(E)$ is the $20 \times 20$ subspace Hamiltonian for the $s, p_{x}, p_{y}$, $p_{z}$, and $s^{*}$ orbitals (Ref. 13) on the surface Ga, surface As, subsurface $\mathrm{Ga}$, and subsurface As atoms in the first two planes at the relaxed (110) surface of GaAs (Ref. 2), and the $E_{i}(E)$ are its eigenvalues. The contour in the energy plane runs from $(-13,0)$ to $(-13,10)$ to $(1.6,10)$ to $(1.6,0)$, where the first number in parentheses is the real part of $E$, the second is the imaginary part, and both are given in $\mathrm{eV}$. Notice that $\operatorname{Re} E_{i}(E)$ is nearly constant around the contour until the real axis is approached at $E=1.6$ (an energy very near the conduction-band edge $E_{c}=1.55$ $\mathrm{eV}$-i.e., very near the Fermi energy $E_{F}$ for degenerately doped $n$-type GaAs). 


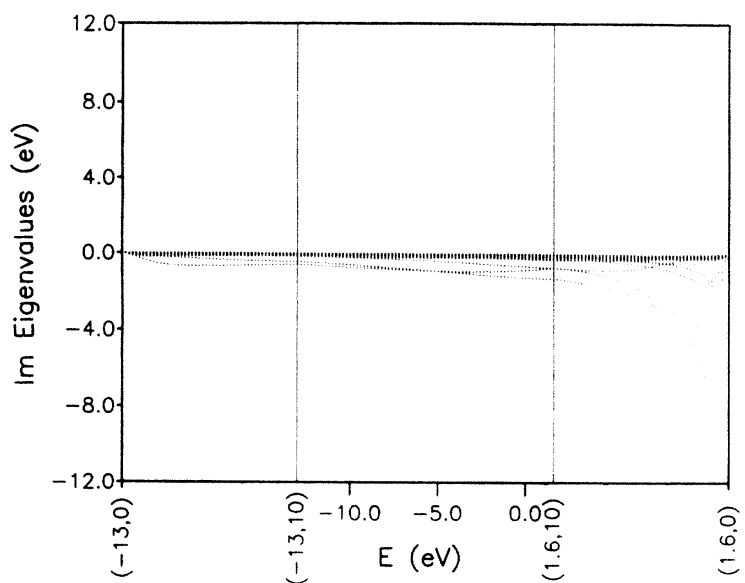

FIG. 2. $\operatorname{Im} E_{i}(E)$ for the same system and same contour as in Fig. 1. Notice that $\operatorname{Im} E_{i}(E)$ is small until the real axis is approached at $E=1.6$. Also notice that $\operatorname{Im} E_{i}(E) \leq 0$.

$\left(C_{2}\right)$, we use a representation like (5.4), with the $E_{i}(E)$, $\psi_{i}(E)$, and $\bar{\psi}_{i}(E)$ calculated for some representative sample of the energies $E$.

Many applications require the Green's function outside the perturbation subspace-i.e., $G^{12}, G^{21}$, and $G^{22}$. These can be obtained from the $G=G^{11}$ of (5.4) by means of the Dyson equation (2.8).

In some application, one needs the time-dependent Green's function $G\left(t, t^{\prime}\right)$ for phonons, electrons, etc. $G\left(t, t^{\prime}\right)$ is an inverse Fourier transform, i.e., it is also given by an integral along the $\omega$ axis. It can therefore be calculated by the methods of this section.

\section{REAL-TIME COMPUTER SIMULATIONS}

Let $x$ represent one component of the position vector for one nucleus in a system of atoms. In order to perform a computer simulation of the motion of the nuclei, we must be able to calculate the forces

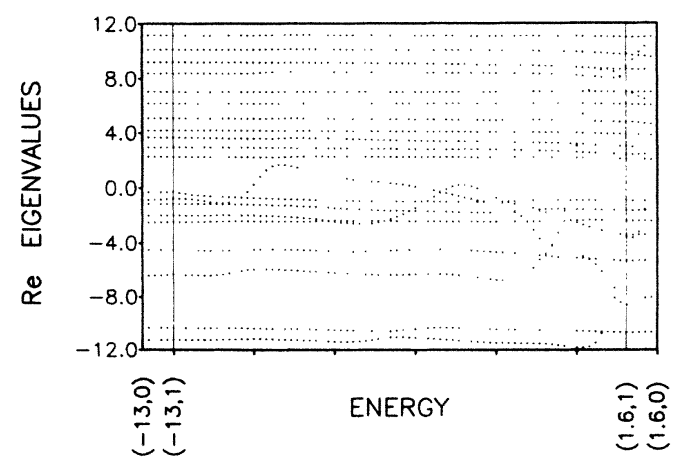

FIG. 3. $\operatorname{Re} E_{i}(E)$ for a contour much nearer the real axis than that of Fig. 1 (with the long segment $1 \mathrm{eV}$ away rather than $10 \mathrm{eV})$. Notice that $\operatorname{Re} E_{i}(E)$ still exhibits a remarkably slow variation with $E$.

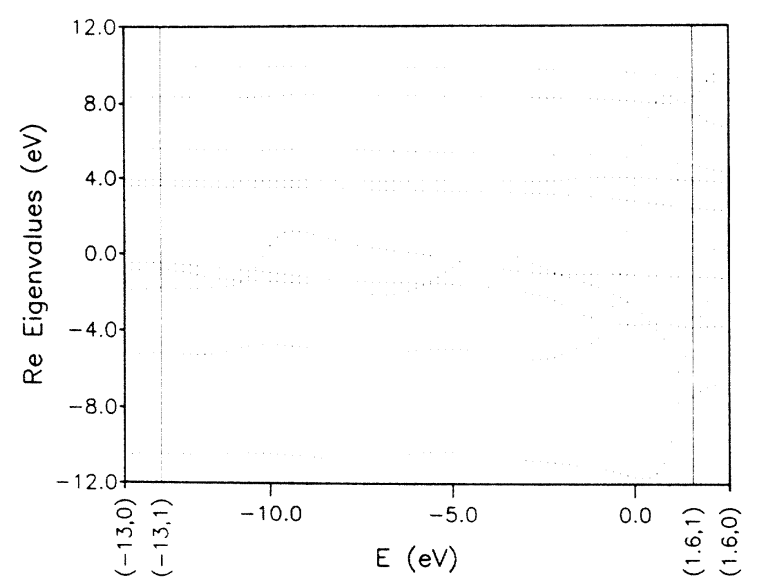

FIG. 4. $\operatorname{Re} E_{i}(E)$ for the same contour and same system as in Fig. 3, but for the $10 \times 10$ subspace Hamiltonian corresponding only to surface $\mathrm{Ga}$ and surface As atoms. Note that $E-H_{\text {sub }}(10 \times 10) \equiv[G(10 \times 10)]^{-1}$, whereas $E-H_{\text {sub }}(20 \times 20)$ $\equiv[G(20 \times 20)]^{-1}$, but there is an obvious correspondence in the relevant eigenvalues $E_{i}(E)$.

$$
F_{x}=-\frac{\partial U}{\partial x} .
$$

One contribution to the total energy $U$ is the energy $\Delta E$ of the preceding section. For simplicity, we will not consider the other contributions in the discussion here. Some of these contributions (e.g., the nucleus-nucleus Coulomb repulsion) can be simply treated, and some of them (e.g., the doubly counted electron-electron interaction) can be treated using ideas similar to those presented below.

If we now regard $F_{x}$ as the contribution to the total force arising from the electronic energy $\Delta E,(6.7)$ and (6.12) give

$$
F_{x}=\frac{1}{\pi} \operatorname{Im}\left(\sum_{i} \int_{C} \frac{d E}{E-E_{i}(E)} \frac{\partial E_{i}(E)}{\partial x}\right) .
$$

According to the arguments of the preceding section,

$F_{x}=F_{1}+F_{2}$,

$$
\begin{aligned}
& F_{1} \approx \frac{1}{\pi} \operatorname{Im}\left[\sum_{i} \frac{\partial E_{i}\left(E_{0}\right)}{\partial x} \ln \left[\frac{E_{a}-E_{i}\left(E_{0}\right)}{E_{c}-E_{i}\left(E_{0}\right)}\right)\right], \\
& F_{2}=\frac{1}{\pi} \operatorname{Im}\left[\sum_{i} \int_{C_{2}} \frac{d E}{E-E_{i}(E)} \frac{\partial E_{i}(E)}{\partial x}\right] .
\end{aligned}
$$

Here, $E_{a}$ is the complex energy where $C_{1}$ stops and $C_{2}$ begins. According to the Helmann-Feynman theorem [compare with (3.3)],

$$
\frac{\partial E_{i}(E)}{\partial x}=\bar{\psi}_{i}^{\dagger}(E) \frac{\partial V}{\partial x} \psi_{i}(E)
$$

since $\partial H_{\text {sub }} / \partial x=\partial V / \partial x$ in view of (2.12). Very recently, we have successfully employed (7.4) and (7.5) in real-time computer simulations of $\mathrm{Al}$ and As atoms interacting with the (110) surface of GaAs. ${ }^{6}$ 


\section{CONCLUSION}

The mathematical and numerical results of this paper help to explain previous successes of the subspace Hamiltonian technique in practical calculations, and also help to provide a foundation for a wider variety of applications. This technique promises to be a useful addition to the repertoire of Green's-function methods in physics. ${ }^{16-32}$

\section{ACKNOWLEDGMENTS}

We thank the U.S. Office of Naval Research for their support, which made this work possible (under Contract No. N00014-82-K-0447). Additional support was provided by the Robert A. Welch Foundation (Houston, TX). We benefited from discussions with O. F. Sankey, M. A. Bowen, and A. C. Redfield.
${ }^{1}$ R. E. Allen (unpublished). (See Refs. 2-6.) Although defined in terms of Green's functions [see Eqs. (2.4) and (2.12)], the subspace Hamiltonian can be shown to be mathematically identical to the energy-dependent effective Hamiltonian of Löwdin and Pryce. That is, $H_{\text {sub }}(E)=H^{11}+\Sigma(E)$, $\Sigma(E)=H^{12}\left(E-H^{22}\right)^{-1} H^{21}$, in the notation of Eq. (4.2), with $E$ in the last expression regarded as multiplied by the $(N-n) \times(N-n)$ identity matrix of the 2 subspace. See P. O. Löwdin, J. Chem. Phys. 19, 1396 (1951); M. H. L. Pryce, Proc. Phys. Soc. London 63, 25 (1950); A. M. Stoneham, Theory of Defects in Solids (Clarendon, Oxford, 1975), Appen$\operatorname{dix} 2$.

${ }^{2}$ R. P. Beres, R. E. Allen, and J. D. Dow, Phys. Rev. B 26, 769 (1982); 26, 5702 (1982); Solid State Commun. 45, 13 (1983).

${ }^{3}$ T. J. Humphreys and R. E. Allen (unpublished).

${ }^{4}$ H. Lim and R. E. Allen, J. Vac. Sci. Technol. B 3, 1221 (1985).

${ }^{5}$ S.-F. Ren and R. E. Allen, Surf. Sci. 148, 2637 (1984).

6M. Menon and R. E. Allen, in Proceedings of the 1985 Summer Computer Simulation Conference (North-Holland, Amsterdam, 1985), p. 219.

${ }^{7}$ R. E. Allen, Phys. Rev. B 20, 1454 (1979).

${ }^{8} \mathrm{G}$. Baym, Lectures on Quantum Mechanics (Benjamin, Reading, Mass., 1981), pp. 106-107.

${ }^{9}$ B. S. DeWitt, Phys. Rev. 103, 1565 (1956).

${ }^{10}$ G. L. Toulouse, Solid State Commun. 4, 593 (1966).

${ }^{11}$ J. Friedel, Ann. Phys. (Paris) 9, 158 (1954).

${ }^{12}$ D. C. Langreth, Phys. Rev. B 5, 2842 (1972).

${ }^{13}$ P. Vogl, H. P. Hjalmarson, and J. D. Dow, J. Phys. Chem. Solids 44, 365 (1983).

${ }^{14}$ Calculations of the bulk Green's function $G_{0}$ and the surface Green's function $G$ were performed exactly as in Refs. 2 and 5. The results of Figs. 1-4 are for the special point, in the center of the irreducible $\frac{1}{4}$ th of the surface Brillouin zone.
${ }^{15}$ O. F. Sankey (unpublished).

${ }^{16}$ I. M. Lifshitz, Nuovo Cimento Suppl. 3, 716 (1956).

${ }^{17}$ G. F. Koster and J. C. Slater, Phys. Rev. 96, 1208 (1954).

${ }^{18}$ M. Lannoo and P. Lenglert, J. Phys. Chem. Solids 30, 2409 (1969).

${ }^{19}$ G. Allan, Ann. Phys. (Paris) 5, 169 (1970).

20D. Kalkstein and P. Soven, Surf. Sci. 26, 85 (1971).

${ }^{21}$ T. L. Einstein and J. R. Schrieffer, Phys. Rev. B 7, 3629 (1973).

${ }^{22}$ F. D. M. Haldane and P. W. Anderson, Phys. Rev. B 13, 2553 (1976).

${ }^{23}$ A. A. Maradudin, Rep. Prog. Phys. 28, 331 (1965).

24J. Callaway, Quantum Theory of the Solid State (Academic, New York, 1974), Chap. 5.

${ }^{25}$ M. Lannoo and J. Bourgoin, Point Defects in Semiconductors I. Theoretical Aspects (Springer-Verlag, New York, 1981).

${ }^{26}$ E. N. Economou, Green's Functions in Quantum Physics (Springer-Verlag, New York, 1979).

${ }^{27}$ G. A. Baraff and M. Schlüter, Phys. Rev. Lett. 41, 892 (1978).

28J. Bernholc, N. O. Lipari, and S. Pantelides, Phys. Rev. Lett. 41, 895 (1978).

${ }^{29}$ L. P. Kadanoff and G. Baym, Quantum Statistical Mechanics (Benjamin, Menlo Park, Calif., 1962).

${ }^{30}$ A. A. Abrikosov, L. P. Gor'kov, and I. E. Dzyaloshinski, Methods of Quantum Field Theory in Statistical Physics (Prentice-Hall, Englewood Cliffs, NJ, 1963).

${ }^{31}$ A. L. Fetter and J. D. Walecka, Quantum Theory of ManyParticle Systems (McGraw-Hill, New York, 1971), and refereces therein.

${ }^{32}$ S. Doniach and E. H. Sondheimer, Green's Functions for Solid State Physicists (Benjamin, Reading, Mass. 1974), and references therein. 